\title{
First assessments by specialist cancer nurses in the community: an ethnography
}

Elizabeth Gamlen, Community Macmillan Nurse Specialist, The Beacon Centre, Birgin Care Ltd., Guildford Surrey G22 7WW

Anne Arber, Duke of Kent Building, University of Surrey, Guildford, GU2 7TE 


\begin{abstract}
Purpose: The aim of the study is to explore how specialist cancer nurses carry out first assessments of patients in the community, their use of the Symptoms and Concerns Checklist (SCC) and their views on first assessments.

Methods \& Sample: An ethnographic approach was taken. The data were collected by use of non-participant observation of specialist nurses carrying out a first assessment of patients with cancer, followed by semi-structured interviews with six specialist nurses. Data were analysed using iterative thematic analysis.

Key results: All the specialist nurses recognised the value of facilitating the patients' narrative. Use of the SCC was seen to jar with the overall theme of building relationships during the first assessment visit. It was recognised by the specialist nurses that the SCC was beneficial in prompting the patients to discuss psychosocial concerns.

Conclusions: This study gives insight into the conflict and ambiguity for specialist nurses when using the SCC during the first assessment visit. It confirms the SCC as beneficial in cueing some patients to identify specific concerns and for these concerns to be recognised by the specialist nurses.
\end{abstract}

Key words: First assessments, cancer, community, specialist cancer nurses, ethnography, Symptoms and Concerns Checklist. 


\section{Introduction}

Specialist nurses use many different methods to assess and elicit patient problems and a number of different assessment tools are used in practice ew studies have focused on the process of carrying out first assessments by specialist nurses in the community. However, Hunt (1991), carried out a ground-breaking study of conversations between terminally ill patients and specialist nurses. Hunt found that specialist nurses used their interpersonal skills to present themselves as 'friendly and informal' during conversations with patients; this enabled the patients and nurses to break down barriers so that trust developed and patients concerns were elicited. However this approach also enabled the nurses to keep control of the flow of topics and questions asked. Few studies have looked at how interpersonal and relationship building skills are used in conjunction with the use of a validated assessment tool (Wilkinson et al., 2003).

Advocates of structured assessment tools emphasise how they are to be used in partnership with the patient who can be talked through a list of questions, prompts and triggers, which help the patient to think about their own needs (Cowley et al., 2004). Little is known about professionals' views and experiences of using assessment tools and some negative views have been reported about their use (Hughes et al., 2003; Baba et al., 2007). Issues found to be important when implementing assessment tools were time constraints, staff workload, training and the potential burden on the patient (Hughes et al., 2003; Dunckley et al., 2005). However use of such tools provides a base-line assessment of individual patient need (Department of Health, 2002).

In a national scoping exercise of the 34 cancer networks in England Richardson et al., (2005) found that $67 \%$ of the cancer networks sampled used at least one assessment tool. Assessment tools were found to be adopted if they met the criteria of ease of administration, acceptability to patients, validity, and compatibility or were established in practice. However, no tool has been identified as the gold standard for a nursing assessment (Richardson et al. 2006).

One of the assessment tools used in practice is called the Symptoms and Concerns Checklist (SCC) a validated assessment tool developed by Lidstone et al., (2003). The SCC consists of 29 items designed to be completed by patients. The aim of the SCC is to enable prioritisation of concerns and speedy access to specialist palliative 
care services for those with advanced cancer. Fifteen items on the SCC relate to physical, psychological or cognitive problems. The remaining 14 items relate to selfcare, relationships, financial issues, work and the future; thus demonstrating to patients significant areas that a specialist nurse would be interested in.

\section{Aims of the study}

This study investigates specialist nurses' first visits to patients with cancer in the community. The study also explores how specialist nurses use the SCC in practice and their views on the SCC.

\section{Method}

An Ethnographic approach was selected as the research method for the study. This approach combines non-participant observation of first visits by specialist cancer nurses to patients in the community followed by semi-structured interviews with the specialist nurses. The ethnographic approach enabled the researcher, who is also a specialist cancer nurse, to question the familiar (Lambert and McKevitt, 2002) and to provide a 'thick' description of how the first assessment was carried out and to hear specialist nurses' views on the impact of using the SCC.

The naturalistic approach using ethnography regards the knowledge and practice of 'experts' as locally variable rather than assuming that biomedical concepts and practices are universal (Lambert and McKevitt, 2002). A purposive sample of seven specialist cancer nurses experienced in using the SCC were invited to participate in the study and six came forward to participate. The specialist nurses were all female, aged between 40-50 years. They had been specialist nurses from between two and seven years and had prior experience in oncology of between two and ten years. So they were all experienced specialist nurses with a minimum of two years experience in the field.

Data were collected from 6 non-participant observation episodes followed by 6 semistructured interviews with specialist nurses following the first assessment visit. The first assessments in this study represented non-urgent referrals to the service. Three methods of recording data were used: non-participant observation and the writing of field notes; semi-structured interviews, which were audio recorded as a means to further illuminate the use of the SCC and uncover specialist nurses views of the 
SCC; and finally a field journal was kept to reflect on the research as it unfolded and to document how the specialist nurses were reacting to the researcher. The research journal helped the researcher to reflect on her insider (specialist nurse) and outsider (researcher) status and to integrate thinking and avoid fragmenting of the data and to keep a footing as a researcher. Credibility and rigour were maintained by use of the research journal, by the use of thick description within the field notes and by keeping an audit trail including regular interaction with the research supervisor and avoidance of 'going native' as a practitioner rather than a researcher (O'Reilly, 2008).

The researcher travelled to the patient's home with the specialist nurse. The first assessment was observed to take between $45 \mathrm{~min}$ and $1 \mathrm{~h} 15 \mathrm{~min}$. The field notes were recorded at the time of the assessment while sitting with the specialist nurse and the patient. The semi-structured interviews with the specialist nurse were conducted with the same nurse as soon as it was possible following the observed episode. The interview was audio-recorded and lasted between 25 and $35 \mathrm{~min}$ in length. An interview schedule (topic guide) was used which consisted of an opening question: 'How did you feel the assessment went? followed by other open-ended questions about the first assessment. The audio-recordings were transcribed verbatim as soon as possible after the interview.

Data from the semi-structured interviews and field notes were analysed using iterative thematic analysis. This approach stems from a tradition of qualitative research where the researcher is guided by the data rather than a pre-established hypothesis (Hansen, 2006; Silverman, 2012). Throughout the period of data collection the researcher was immersed in the data as it arose through nonparticipant observation and the writing of field notes. A process of colour coding the data was employed highlighting interesting aspects, assigning parts of the data to codes for future retrieval and then assigning them to emerging themes (Hammersley and Atkinson, 2007; O'Reilly, 2008). The researcher became familiar with the data in its entirety by reading and re-reading the transcripts and field notes. The research journal enabled the researcher to reflect on the emerging themes and to begin to refine and check the themes for completeness. Ethical approval for the study was granted by the National Research Ethics Committee. 


\section{Results}

All the specialist nurses recognised the value of facilitating the patient's story and this was the overarching theme that was identified. Three other themes that emerged from the data are called: threats to trust and relationship building, timing of the SCC alongside the patient's story and using the SCC to validate the patient's story.

\section{Facilitating the patient's story}

The specialist nurses were observed to facilitate the patients to tell their story as a way of hearing about the patient's current and past situation. The specialist nurses described how understanding these stories would give insight into the patient's experience. N4 reports how she observes how the patient tells their story and the words they use:

N4: I like to hear how a person says their story in their own words because their story.... because I think obviously their story and their words tell me what they understand but also what words they use and whether there's anger there or ..um ..denial, any of their coping mechanisms that may be shutting down really.

N4 believes hearing the patient's story will inform her about how the patient constructs the meaning of their experience, and what the patient understands about the situation. Most importantly she looks for signs of how the patient is coping and the patient's emotional expression in terms of the psychological mechanisms of denial and anger.

N6 reports how hearing the patient's history and story and talking with the patient is very important:

N6: what helps is a history and what helps is talking to the patient, listening to what they're saying as well as what you're seeing and, if for some reason that patient isn't capable of telling you their story or talking to you then I think you're really working in the dark and you've got to rely on other professionals and their opinions which is going to be a bit harder.

In this instance the specialist nurse identifies the importance of getting the history from the patient and hearing the story first hand rather than through a secondary 
source. N6 feels she will be disadvantaged without hearing the patient's own version of their experience. The story is used as a framework by the nurse with which to understand the patient. The alternative will be to use a 'framework' of other health care professionals which is not the same as hearing it directly from the patient.

N4 also describes the patient's story and how when telling his story the patient cried. Therefore the patient was able to express his grief and she describes how: 'his stories very sad, he's very young for a horrible disease'. Thus hearing the patients story enabled the patient to unburden his sorry and to express his emotions and grief.

\section{Threats to trust and relationship building}

Within the first assessment patients are given the time and space to tell their story and to express their grief and this helps with developing trust:

N5: 'I just want to get them on board and to feel as if they can trust me and they feel safe with me and I wouldn't put anything in jeopardy of that. I don't want anything to upset that, especially on that first assessment visit'.

There was concern expressed by N5 that use of the SCC could compromise the relationship building process and could potentially be a barrier to building trust, "being too much' on a first visit, by changing the dynamics of the embryonic relationship.

N3 reports how using the SCC has the potential to cause a distraction and impede the 'flow' in the work of 'building a relationship'. Rather than enabling the relationship process, the SCC was perceived negatively by N3 as 'a bit of paper' which was going to 'bombard' the patient with a burden:

N3: 'I normally like to sort of ask the patient verbally and let them verbalise their concerns rather than handing them a bit paper and say 'tick the boxes', I much prefer to sort of ask.... .rather than bombard them'.

This nurse expressed a preference for using an informal approach to information gathering. She describes the paper work as a barrier to the patient expressing their concerns. She is concerned about making too many demands on the patient related to form filling. She is more interested in facilitating the verbal expression of the 
patient's story rather than the SCC paper work, which similar to N5 was viewed as overly bureaucratic.

The SCC appeared to be a threat to the relationship building process in that it was seen as bureaucratic and impersonal:

N5: 'people fill in so many forms these days and paperwork. Some people actually turn round and say 'not another form' and that puts me off straight away. I think this interview is supposed to be about something else, we're going on a, for me, its going on a journey together, exploring about where they've come from and where they're at and where they're going and we're doing that together. It's not as harsh as filling out a form....coming back to this trust and building relationship I don't want them to remember me as being a burden - I want that first visit to be helpful to them'

N5 is concerned that the SCC represented 'harshness' and may damage her presentation as a 'nice' professional by being seen as a 'burden' to the patient, especially when the patient objects to having another form to fill in. The specialist nurses wish to facilitate the patient's story and to connect on their journey together. The SCC is perceived as a bureaucratic burden by N3, N5 and N6 and a barrier to relationship building and building rapport.

\section{Timing of the SCC alongside the patient's story}

During the fieldwork it became apparent that getting the timing right in administering the SCC was important. N1 refers to giving the SCC to the patient only after she'd spent considerable time listening to the patient:

N1: ' hopefully by then they feel quite at ease, because a long form might um feel,ooh and some of the questions, so hopefully by the time I give them that they feel it is alright and so miss out what they feel uncomfortable with'.

Here $\mathrm{N} 1$ is more concerned for the patient to feel at ease than to complete a long form full of questions. However she is careful in the timing of the SCC so that it is used once the patient is feeling at ease and comfortable. 
N6 also introduced the SCC after hearing the patient's story and reports never starting the assessment with the SCC:

N6 'I never do it straight away. I've tried doing it straight away and it makes my assessment more disjointed.... If I get them to do it first I get too caught up in going through the questions, 'have you got pain?' and all of that , and if l've not already got that history in my head about where they've been, and how they are at that moment, I don't find it very helpful'.

Here N6 is describing how she has experimented with the tool to find the best way to use it. She reports how she prefers to get the patient's story and history clear in her mind before using the SCC as she thinks that introducing the SCC too early may make the story telling aspect and her assessment more disjointed.

One participant thought disclosure was likely to be more comprehensive as a consequence of the rapidly developing therapeutic relationship rather than use of the SCC:

Interviewer: 'do you think the tool is helpful in raising difficult aspects or difficult issues?

N2: 'um possibly, I don't really know about that because sometimes by the time the mere fact they've come up here and have someone listening to them, the difficult issues emerge and they may not necessarily put them down on paper'.

$\mathrm{N} 2$ thought that by agreeing to the assessment (i.e. the patient accepted the invitation to see the nurse) this agreement alone will encourage patient disclosure of concerns and worries. N2 describes her own role in listening to the patient as paramount to engaging with the patient's issues and that the SCC is seen as secondary to this process of engaging.

One of the nurses (N1) found some of the questions on the form to be too personal these concerned sexuality and intimacy and she thought they were too much for the 'older generation'. N1 feels uncomfortable with some of the more intimate questions on the SCC.

The SCC was seen as mechanistic and bureaucratic at the first meeting with the patient where building rapport and trust was a central concern. The specialist nurses handled this conflict by purposefully delaying the use of the SCC and introducing it 'at the right time' when rapport and trust and story telling had been achieved. 


\section{Using the SCC to validate the patient's story}

The SCC is reported by N4 to offer validity to a patient's story by means of a 'check back' mechanism that validates what the patient has already said:

N4: then invite them to tell me their story, and then SCC and then that validates to some degree what they've said in their story, as I felt it did do, yeah, I think the tool really just brings out a lot of what we've really talked about but it kind of consolidates it like, "so you ring (on the tool) pain, so lets talk about the pain"'

$\mathrm{N} 4$ is explaining how the SCC can create agreement between listening to the patient's story and comparing this with what is marked on the SCC. N4 describes the usefulness of the SCC by using it to find out more from the patient about problems such a pain and enables N4 to address the patient's specific problems. N4 sees the usefulness of the SCC as a form of consolidation of the assessment process.

N3 identifies how the patient identified fatigue as a key issue:

N3: 'there is just the one ' 3 ' which is the fatigue: was the only one that scored highly, everything else was, which really reflected what she was saying, you know, she was feeling ok, and the only thing which reflected on the chart was the fatigue'.

The patient's high score of 3 in the SCC identified her key problem and this was supported in the N3's view by what the patient had just told her. Similarly N2 was observed going through the SCC checking out specific problems such as pain, nausea, constipation, sleep pattern, breathlessness and low mood:

'N2: now, looking at your form, so you have no problems with pain or nausea or vomiting?

$P 2$ : but I have problems with constipation and my urine

N2: asks questions re constipation and asks for clarification. Then refers back to SCC and asks her about her sleep.

P2: describes her poor sleep pattern and N6 nods sympathetically

N2: you say you also get breathlessness (referring to SCC) - do you smoke?

P2: I've tried hard to stop - l've cut it down 
N2: you've marked concentration and remembering things? You've also said you feel very low in your mood?

P2: yes- an all time low

This opens up a long discussion about the history of her anxiety.....'

Field notes $\mathrm{P} 2 / \mathrm{N} 2$

N2 was observed to make links with what the patient had already disclosed and the SCC. N2 asked for more information about P2's low mood, which was identified as a serious problem on the SCC. Used in this way the SCC was observed to facilitate $\mathrm{N} 2$ and P2 to explore the serious impact of low mood, anxiety and depression.

One patient was observed to deny having any concerns when asked directly by the specialist nurse but the patient then went on to mark 16 out of 29 items on the SCC as being significant problems:

N3: she...sort of said 'well I haven't got any [concerns]', which is why I thought by giving her the sort of concerns aide memoir [SCC] to see whether that would help her to identify.

The SCC was used by N3 when she found the patient was unable to identify any concerns and the patient was not able to tell her story. Therefore the SCC is considered by N3 as something to use in this situation as an 'aide memoir'. Perhaps the word 'concerns' was difficult for the patient to identify with and the more concrete items on the SCC helped to cue the patient and establish her problems.

The SCC prompted nurses to enquire about spirituality and sexuality and 'nonphysical' domains as part of a holistic assessment. All the specialist nurses agreed that the SCC prompted them to conduct a comprehensive and holistic assessment especially the psycho-social domain:

N1: 'So I think that its still helpful because it [SCC] makes you actually go there and ask, had you not thought about it, especially with non-physical stuff, which may be, we sometimes forget .'

$\mathrm{N} 1$ is identifying the primary importance of the physical assessment of patients. She uses 'we' rather than ' $l$ ' to state that the 'non-physical stuff' can sometimes be overlooked and is one of the reasons the SCC was introduced in the first place. The SCC indicated to patients that the specialist nurses were interested in psycho- social 
and spiritual domains. It cued nurses and patients to talk about emotional and psycho-social issues as well as physical problems and it communicated to patients that the specialist nurse was interested in a wide range of concerns.

\section{Discussion}

The specialist nurses are ambivalent about using the SCC. They have concerns about using a tool that reduces the assessment to phenomena that are entirely measurable that could hinder the expression of human caring values and their quest for the patient's story. While such reductionism was neither observed nor referred to during the study participants did experience tension between building the relationship on the first visit and using the SCC. In particular the SCC was thought by some to jar with the need to build trust and rapport. All the specialist nurses were careful about the timing of using the SCC and it was always used after hearing the patient's story. Only on one occasion when the nurse had difficulty in getting the patient to tell her story was the SCC used early on in the assessment and this proved successful in cueing the patient to talk about her concerns.

The use of the SCC challenges specialist nurses during the first assessment as they see it as having the potential to interrupt the flow of the assessment and to disturb rapport building with patients. Li (2004) discusses how palliative care nurses and patients develop mutually collaborative 'nice' talk that serves to maintain some order and inspire confidence by patients in the care providers. Her study offers a reflection on how use of an assessment tool like the SCC may take some of the 'order' and safety from both parties by touching on domains of psychosocial care that gives voice to the devastating effects of living with cancer that social convention dictates should not be discussed so soon in a relationship. However, the discomfort some of the specialist nurses described regarding use of the SCC may be partly explained by Hartrick (1997) who identifies how a medical/technological paradigm continues to exert profound influence on interpersonal nursing practice. Thus using the SCC may conflict with nurses' beliefs and values about how to express human caring values, which are not consonant with using a checklist approach and scoring system when assessing patients during sensitive first meetings.

The specialist nurse participants all referred to the benefits of the SCC as a prompt or 'aide memoire' to enquire about the psycho-social impact of cancer, and this benefit was observed as facilitating the patients to report these issues. The specialist 
nurses acknowledged that the SCC could fulfil this function and literature suggests patients are keen that health care professionals enquire about psycho-social issues in their lives (Cull et al., 1995; Irving et al., 2004; Whelan et al., 1997; Hill et al., 2003). Patients vary greatly in their ability to voice their concerns therefore it is believed that communication will be enhanced by a comprehensive and holistic enquiry into patients needs (Crooks et al., 2004). Slater and Freeman, (2004) demonstrated how patients were relieved to record their psychological distress, especially if it were a sensitive concern i.e. with their family member. The action of writing their psychological concerns down was described as 'comforting'.

The specialist nurses were concerned about the timing of use of the SCC and had concerns about some of the items on the SCC and wished to avoid causing additional distress to patients (Hopkinson et al., 2006; Hughes et al., 2003). Patients may feel violated if they have shared sensitive information prematurely or against their wishes (Widang and Fridlund, 2003). Slater and Freeman, (2004) found that some patients were initially angry about being asked about their anxiety regarding treatment, whereas for some this was an opportunity to express their frustration arising from debilitating treatment side-effects, identifying the importance of nurses continuing to individualise their approach to each patient. Interestingly in the same study patients were critical of the assessment tool finding it too problem focussed and that it didn't allow the patient to report the positive things they had achieved. This is an interesting point that was not raised in this study and supports the argument for the SCC being offered within the context of an assessment that allows a timely articulation of the narrative and personal priorities and agendas of the patient.

The specialist nurses were least comfortable asking questions around the patients' irretrievable losses. Sexual, financial, and spiritual matters are recognised as difficult for health care practitioners to enquire about (Crooks et al., 2004) because it can feel an unacceptable intrusion (Dunckley et al., 2005). The SCC makes provision for a patient to self-score their concerns regarding sexual or spiritual issues but there is disquiet described in the literature and shared by the participants in this study about asking about these domains (Heaven and Maguire, 1998). However it is reported that some patients with cancer wish to discuss the impact of disease on sexual function and see this as important aspect of their quality of life (Ananth et al., 2003; Osse et al., 2002). The extent to which patients wish to have their spiritual needs incorporated into their initial assessment is unclear. Spiritual care recognises the relationship between illness and the spiritual domain and searching for meaning 
around illness and the future. Some patients have been found to actively seek to disguise their spiritual distress (Mclllmurray et al., 2001), which is one conclusion that could be drawn from the lack of spiritual concern identified by patients when filling in the SCC in the course of this study. Richardson et al., (2006) suggest caution in relation to use of an assessment tool when considering the spiritual well-being domain as they suggest that this type of self-assessment may not be the best way for assessing this domain. A more phased assessment of 'personal domains' such as sexuality and spirituality undertaken in an iterative mode has been identified as preferable (Murray et al., 2004; McSherry and Ross, 2002).

For the future the introduction and implementation of an assessment tool should be embedded within the total assessment process. For example training in Advanced Communication Skills should include how to incorporate such tools without impeding the flow of the assessment, the development of rapport, trust and relationship building. The barriers to using such tools in terms of specialist nurse attitudes need to be considered. Evidence of the effectiveness of the tools such as the SCC in terms of patient outcomes are needed as well as a consideration of training needs when incorporating these tools into practice (Hughes et al., 2003; Slater and Freeman, 2004).

\section{Conclusion}

The findings of this study indicate the complex nature of the assessment phenomenon. The over-arching aims of the specialist nurses during the first assessment were to build relationships and rapport with their patients. They preferred to use the patients' narrative as a means to develop this connection and carry out a holistic assessment. This study gives insight into the potential conflict of purpose for these specialist nurses as they undertake a first assessment and offers guidance on implementing assessment tools such as the SCC in the future. The potential for open and honest regard for the patient as an individual appears to be possible by using the SCC in a timely way and within the relationship and trust building process. While the use of the SCC will not replace intuitive and specialist skills it has the potential to help patients and professionals identify individual needs and quite possibly enhance the therapeutic relationship.

\section{Conflict of interest}

None declared 


\section{References}

Ananth, H., Jones, L., King, M., Tookman A., 2003. The impact of cancer on sexual function : a controlled study. Palliative Medicine 17, 202-205.

Baba, K., Fransson, P.,Lindh, J., 2007. Use of a modified ESAS in cancer patients: a pilot study of patient and staff experiences. International Journal of Palliative Nursing 13, 610-616.

Cowley, S., Mitcheson, J., Houston, A., 2004. Structuring health needs assessments: the medicalisation of health visiting. Sociology of Health and Illness 26, 503-526.

Crooks, D., Whelan, T., Reyno, L., Willan, A., Tozer, R., Mings, D.,Miller, J.,Tew,M., Elliot, P., Levine, M., 2004. The initial health assessment : an intervention to identify the supportive care needs of cancer patients,. Support Care Cancer 12,19-24.

Cull, A., Stewart, M., Altman, D., 1995. Assessment of intervention for psychosocial problems in routine oncology practice. British Journal of Cancer 72, 229-235.

Department of Health (2002) Guidance on the single assessment process for older people: Health Service Circular. London.

Dunckley, M., Aspinal, F., Addington-Hall, J., Hughes, R., Higginson, IJ., 2005. A research study to identify facilitators and barriers to outcome measure implementation. International Journal of Palliative Nursing 11, 218-225.

Hammersley, M., Atkinson, P., 2007. Ethnography - principles in practice. $3^{\text {rd }}$ Edition. Routledge, London.

Hansen, E., 2006. Successful Qualitative Health Research - a practical approach. Open University Press, Milton Keynes.

Hartrick, G., 1997. Relational capacity :the foundation for interpersonal nursing practice. Journal of Advanced Nursing 26, 523-528.

Heaven, C., Maguire, P. 1998. The relationship between patients' concerns and psychological distress in a hospice setting. Psycho-oncology 7, 502-507.

Hill, K., Amir, Z., Muers, M., Connolly, C., Round, C., 2003. Do newly diagnosed lung cancer patients feel their concerns are being met? European Journal of Cancer Care 12, 35-45.

Hopkinson, J., Wright, D., Corner, J., 2006. Exploring the experience of weight loss in people with advanced cancer. Journal of Advanced Nursing 54, 304-312.

Hughes, R., Aspinal, F., Addington-Hall, J., Chidgey, J., Drescher, U., Dunckley, M., Higginson, I., 2003. Professional's views and experiences of using outcome measures in palliative care. International Journal of Palliative Nursing 9, 234-238.

Hunt, M., 1991. Being friendly and informal: reflected in nurses', terminally ill patients' and relatives' conversations at home. Journal of Advanced Nursing 16, 929938. 
Irving, M., 2004. Developing a self-assessment quality of life tool. Cancer Nursing Practice 3, 33-39.

Lambert, H., McKevitt, C., 2002. Anthropology in health research: from qualitative methods to multidisciplinarity. British Medical Journal 325, 210-213.

Li, S., 2004. 'Symbiotic niceness': constructing a therapeutic relationship in psychosocial palliative care. Social Science and Medicine 58, 2571-2583.

Lidstone, V., Butters, E., Seed, P., Sinnott, C., Beynon, T., Richards, M., 2003. Symptoms and concerns amongst cancer outpatients: identifying the need for specialist palliative care. Palliative Medicine 17, 588-595.

Mclllmurray, M., Thomas, C., Francis, B., Soothill, K., Al-Hamad, A., 2001. The psychosocial needs of cancer patients: findings from an observational study. European Journal of Cancer Care 10, 261-269.

McSherry, W., Ross, L., 2002. Dilemmas of spiritual assessment: considerations for nursing practice. Journal of Advanced Nursing 38, 479-488.

Murray, S., Kendall, M., Boyd, K., Worth, A., Benton, T., 2004. Exploring the spiritual needs of people dying of lung cancer or heart failure: a prospective qualitative interview study of patients and their carers. Palliative Medicine 18, 39-45.

O'Reilly, K., (2008) Key Concepts in Ethnography. Sage, London.

Osse, B., Vernooij-Dassen, M., Schade, E., de Vree, B., Muijsenbergh, M., Grol, R., 2002. Problems to discuss with cancer patients in palliative care: a comprehensive approach. Patient Education and Counselling 47, 195-204.

Richardson, A., Sitzia, J., Brown, V., Medina, J., 2005. Patients' needs assessment tools in cancer care: principles and practice. Kings College, London.

Richardson, A., Tebbit, P., Brown, V., Sitzia, J., 2006. Assessment of Supportive and Palliative Care Needs for Adults with Cancer . Kings College ,London.

Silverman, D., 2012. Interpreting qualitative data: methods for analysing talk, text and interaction. $4^{\text {th }}$ edition, Sage, London.

Slater, A., Freeman, E., 2004. Patients' views of using an outcome measure in palliative day care: a focus group study. International Journal of Palliative Nursing 10, 343-351.

Whelan, T., Mohide, E., Willan, A., Arnold, A., Tew, M., Sellick, S., Gafni, A., Levine, M., 1997. The supportive care needs of newly diagnosed cancer patients attending a regional cancer centre. Cancer 80, 1518-1524.

Widang, I., Fridlund, B., 2003. Self respect, dignity and confidence: conceptions of integrity among male patients. Journal of Advanced Nursing 42, 47-56.

Wilkinson, S., Leliopoulou, C., Gambles, M., Roberts, A., 2003. Can intensive three day programmes improve nurses' communication skills in cancer care? Psychooncology $12, .747-759$. 